\title{
The Italian Site for KM3NeT ARCA
}

\author{
Giorgio Riccobene ${ }^{1, *}$ for the KM3NeT Collaboration \\ ${ }^{1}$ INFN-LNS, Via S-Sofia 62, I-95123 Catania, Italy
}

\begin{abstract}
The Italian site for KM3NeT, located in the Ionian Sea about $100 \mathrm{~km}$ offshore Capo Passero, South East of Sicily, is dedicated to host (at least) two building blocks of the ARCA (Astronomy Research with Cosmics in the Abysses), comprising 230 Detection Units aiming at measurement of high-energy neutrino fluxes and discovery of their sources. The existing infrastructure is under upgrade within the framework of the IDMAR project jointly funded by Regione Siciliana and INFN. IDMAR at Capo Passero will be run in connection with the other abyssal infrastructure built by INFN $25 \mathrm{~km}$ offshore the town of Catania at $2100 \mathrm{~m}$ depth, hosting the Western Ionian Sea node of EMSO-ERIC.
\end{abstract}

\section{The Capo Passero Site for KM3NeT}

One of the two KM3NeT nodes (KM3NeT/ARCA) (www.km3net.org) is going to be deployed in the Western Ionian Sea, offshore the Sicilian Coast, about $100 \mathrm{~km}$ offshore the harbour of Capo Passero (Fig. 1). The selected site (covering a surface of about four squared kilometres centered around the coordinates N 36 $16^{\circ} 48.34^{\prime \prime}$, E $15^{\circ} 58^{\prime} 42.25^{\prime \prime}$ ) lies on a wide abyssal plateau at an average depth of $3500 \mathrm{~m}$.

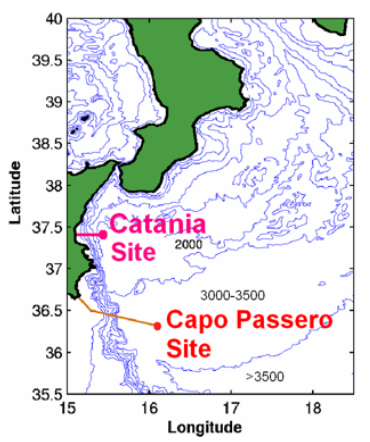

Figure 1. The Abyssal infrastructures realised by INFN offshore Sicily: Capo Passero (3500 m depth) will host KM3NeT/ARCA. The Catania infrastructure is entirely dedicated to multidisciplinary science.

The site has been selected and characterised following a multi-year activity, conducted by INFN, of seek among Mediterranean abyssal sites. Seawater currents have been measured with current meter moorings and show an average value of $3.5 \mathrm{~cm} / \mathrm{s}$ with currents never exceeding $30 \mathrm{~cm} / \mathrm{s}$. Optical properties, measured with several devices, show an absorption

*e-mail: riccobene@lns.infn.it 
length of $70 \mathrm{~m}$ and an effective scattering length $>100 \mathrm{~m}$ for blue light [1]. Optical background shows a small contribution from bioluminescence bursts as demonstrated by the operation of autonomous devices and neutrino telescopes' detection units.

The Capo Passero site is equipped with a Main Electro Optical Cable (MEOC) capable to serve the Phase 1 of KM3NeT/ARCA, and a submarine network of Junction Boxes (JBs) and interlink electro-optical cables. The network is going to be expanded, within the framework of the IDMAR project jointly funded by Regione Siciliana and INFN, with another MEOC and further JBs capable to interconnect the full detector (230 detection units) to shore. A shore lab in the harbor of Portopalo di Capo Passero hosts the MEOC termination(s), the power feeding equipment and the DAQ systems. The shore station will be also refurbished to serve the full ARCA detector. A dedicated optical fiber link , $10 \mathrm{Gbps}$ capability, realised under the GARR-X project (www.garrxprogress.it), connects the harbor station to the Laboratori Nazionali del Sud - INFN (Catania) and, from there to the European Research network backbone, allowing high speed connection from/to the underwater detector controls and data.

During the site search and characterisation activities a large number of measurements have been carried out in collaboration between the astro-particle physics groups and Earth and Sea Science scientists.

A noticeable example is the discovery of abyssal vortexes in the area, revealed by data from current meters and CTD (Conductivity Temperature Depth) [2] which had been recorded by the NEMO Collaboration in multi-year observation campaigns.

The operation of photo-sensors installed onboard a tower-like prototype of detection unit (the NEMO mini-tower), allowed also to study and determine the diel current-driven behaviour of bioluminescence at the Capo Passero Site [3]. The extremely low rates of bioluminescence bursts have been confirmed by the operation of the Prototype KM3NeT Detection Unit [4] and by the first two KM3NeT Detection Units installed at the site in 2015 and 2016. These detectors showed an average photon hit rate of about $7 \mathrm{kHz}$ per each PMT (3" Hamamatsu R12199-02) at single photoelectron threshold, induced mainly by Cherenkov photons from $40 \mathrm{~K}$-decay and dark counts.

Amazingly, colonisation of detector assets (JBs, DU bases) from abyssal macro-fauna has also been observed during ROV footage of the field (Fig. 2, left) and studied [5].

\section{The Catania Test Site}

The potential impact of abyssal observatories designed for neutrino observatories, in the field of Earth and Sea Science is well shown by the operation of the deep-sea infrastructure deployed at $2100 \mathrm{~m}$ depth, $25 \mathrm{~km}$ offshore Catania (see Fig. 1) jointly operated by INFN and INGV. This is the main site of the Western Ionian node of the EMSO-ERIC (https://www.emso.eu) where the NEMO-SN1 seafloor multidisciplinary observatory was put in operation since 2008 [6]. The proximity to the Etna Volcano flanks, Mount Alfeo and the East Sicily shelf break, make this site ideal for geophysics and volcanology studies, attracting research initiatives from several countries: such as the ERC-funded "Focus" project which will monitor movements of the submarine faults close to Etna and the Creep Project (NERC and CSIC) whose goals are the study creeping of rocks in deep sea and the observation of macro-fauna with underwater cameras. The deep-sea site is served by a 28 $\mathrm{km}$ long MEOC, providing direct optical fibre and power connection to four independent terminations on the seafloor, equipped with ROV operable electro-optical connections. Power feeding equipment and DAQ systems are installed on a shore lab in the harbor of Catania, that is also used as construction, assembly and mounting site for deep-sea apparatuses. A spherical pressure tank ( $80 \mathrm{~cm}$ diameter, 400 bar max pressure) is also 
available at the site for tests of the equipment. The shore laboratory has a 1 Gbps link to LNS used for controls and data transfer.

The Catania Site is the first deep-sea real-time passive acoustic monitoring station in the Mediterranean Sea since 2006. NEMO-OvDE [7] was the first acoustic observatory installed at the site, a tetrahedron of $1 \mathrm{~m}$ size of 4 synchronised hydrophones, sensitive in the interval $10 \mathrm{~Hz}-40 \mathrm{kHz}$. Data were digitized off-shore (24 bit, $96 \mathrm{kHz}$ ) and continuously transmitted to shore for analysis. Primarily designed to measure the acoustic noise and its time variation in the aim of a feasibility study for acoustic neutrino detection in the Ionian Sea [8], OvDE revealed itself as a powerful tool for bio-acoustic studies.

Sperm whales were immediately detected and, during two years operation, more than 100 individuals have been identified and their size was determined through passive acoustic methods [9]. The geometrical configuration of the antenna and precise time-stamping, also allows for accurate tracking of the animals and study of their routes (Fig. 2, right).
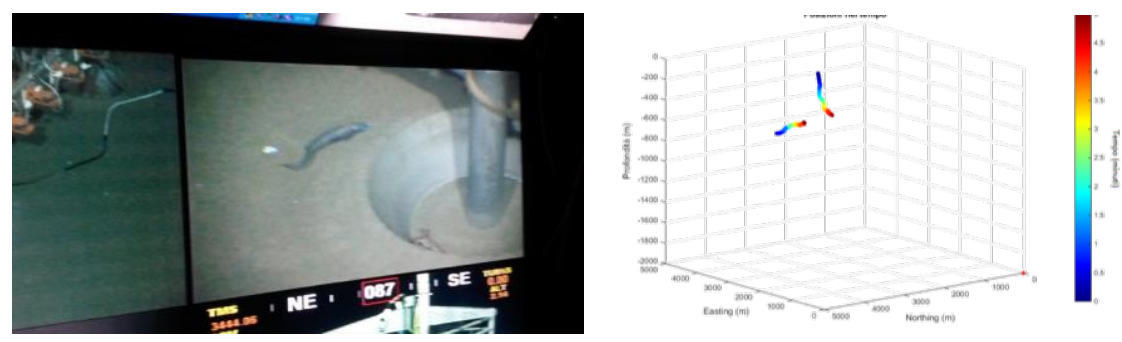

Figure 2. Left: Fishes and crabs colonising a subsea JB of KM3NeT ARCA, observed during ROV video footage. Right:3D-reconstruction (Easting, Northing, depth) of the dive of 2 sperm whales detected by OvDE, The color scale indicate time of detection in a 5 min long acquisition.

The NEMO-OvDE observatory was replaced in 2012 by the SMO-OvDE observatory (Fig. 3, left) that is still running at the site. Realised with similar geometry and general architecture, SMO-OvDE is equipped with very broadband hydrophones $(10 \mathrm{~Hz}-70 \mathrm{kHz})$, and it permits accurate time-stamping of acoustic data acquired off-shore $(<1 \mu \mathrm{s}$ with respect to the GPS time provided from shore).

The observatory continuously monitors the acoustic pollution in the Gulf of Catania and the presence of cetaceans. The detector's frequency band permits also to detect in real-time low frequency signals such as the ones produced by airguns and earthquakes (Fig. 3, right).
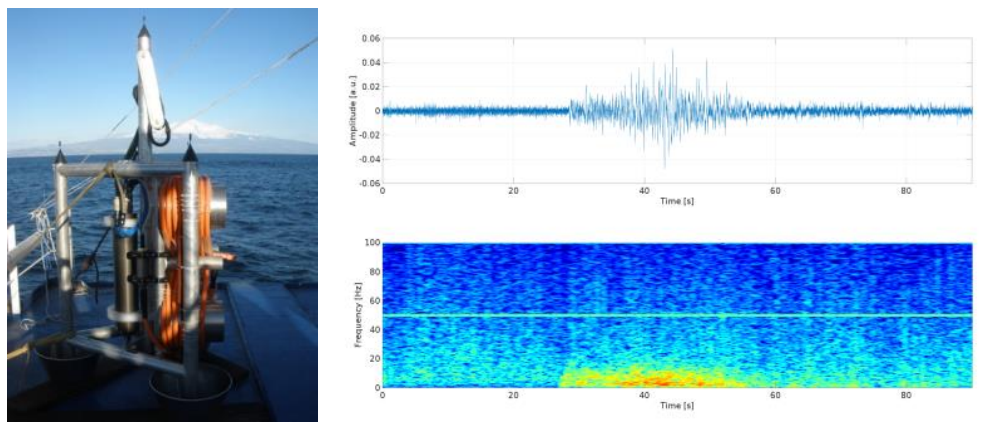

Figure 3. Left: The SMO-OvDE real-time acoustic observatory, deployed in 2012 at $2100 \mathrm{~m}$ depth offshore Catania. The detector is still properly working. Right: Acoustic signature of an earthquake originated by the Etna volcano $(\mathrm{M}=4.8)$, recorded October $6^{\text {th }}$, 2018. Top panel oscillogram, bottom panel spectrogram. 
NEMO-SN1 (Fig.4, left), the first cabled seafloor node of EMSO, was connected to the Catania Test Site infrastructure.
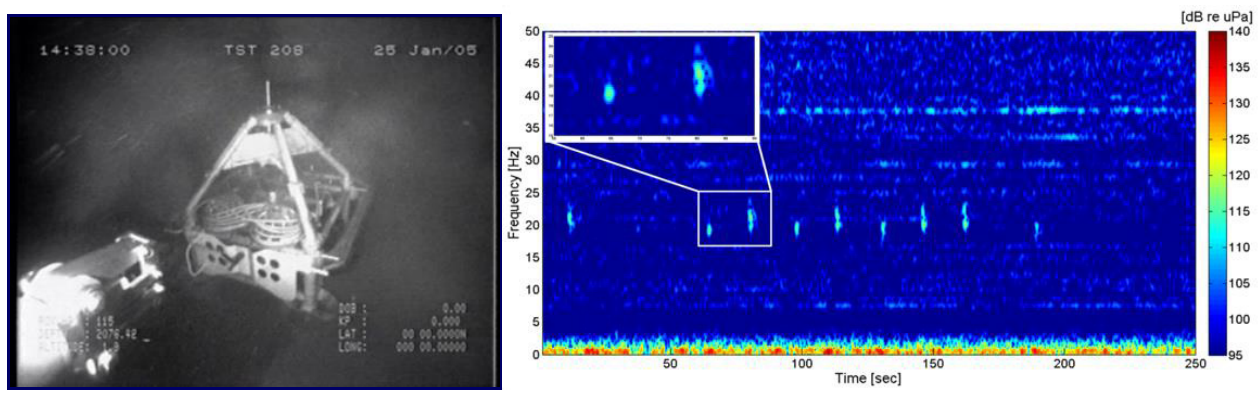

Figure 4. Left: The NEMO-SN1 multidisciplinary observatory deployed at the test site of Catania. Right: Spectrogram of 250 second data showing $18 \mathrm{~Hz}$ fin whale vocalisations (call).

The node is equipped with a three-component broad-band seismometer, a gravity meter, a scalar magnetometer, a three-axes single-point current meter, a CTD and an ADCP, for geophysics and oceanographic monitoring. An array of 4 broadband hydrophones, à la OvDE, was installed on NEMO-SN1 together with a low frequency $(0 \mathrm{~Hz}-1 \mathrm{kHz})$ hydrophone. All sensors are read in real-time from shore.

The low-frequency hydrophone was primarily installed for geophysical research purposes, with the aim of detecting seismic events. It was also extensively used for bioacoustics and acoustic pollution monitoring. Data from this hydrophone permitted to detect (see Fig. 4, right) and analyse the yearly presence of fin whales in the area [11] and measure the acoustic noise in the area. A robust correlation of recorded noise with ship traffic was assessed thanks to data recorded by an AIS antenna installed at the Catania shore laboratory [12]. Acoustic signatures of airgun shootings, produced by ships as far as to close the Greek Coast, were also clearly detected.

\section{References}

1. G. Riccobene et al., Astroparticle Physics 27, 1 (2009)

2. A. Rubino et al., Nature Communications 3, 834 (2012)

3. J. Aguzzi et al., Scientific Reports 7, 44938 (2017)

4. S. Adrian Martinez et al., EPJ C 76, 1 (2016)

5. T.D. Linley et al, 2018, Marine Biology 165, 159 (2018)

6. P. Favali et al., IEEE Journal of Ocean Engineering 38, 358 (2013)

7. G. Riccobene, NIM A 604, S149 (2009)

8. G. Riccobene NIM A 692, 197 (2012)

9. F. Caruso et al., Plos One 10, e0144503 (2015)

10. F. Caruso et al., Scientific Reports 7, 4321 (2017)

11. V. Sciacca et al., Plos One 10, e0141838 (2015)

12. V. Viola et al., Marine Pollution Bulletin 121, 97 (2017) 